\title{
Uso do hábitat e alimentação em juvenis de Bothrops jararaca (Serpentes, Viperidae) na Mata Atlântica do sudeste do Brasil
}

\author{
Paulo Afonso Hartmann, Marília Teresinha Hartmann e Luis Olímpio Menta Giasson \\ Pós-graduação em Ciências Biológicas, Departamento de Zoologia, Instituto de Biociências, Universidade Estadual \\ Paulista, Caixa Postal 199, 13506-900, Rio Claro, SP, Brasil.
}

\begin{abstract}
Habitat use and feeding in juveniles of Bothrops jararaca (Serpentes, Viperidae) in the Atlantic Forest of southeastern Brazil. Herein we describe habitat use, diet, and subjugation behavior of prey in juveniles of Bothrops jararaca that occur in streams in the Atlantic Forest, north coast of the state of São Paulo, Brazil. Additional information on juveniles of $B$. jararacussu found in similar situations to those of $B$. jararaca are also discussed. Individuals of B. jararaca were observed mainly on the ground during the day, and on the ground and also on the vegetation at night. The majority of prey were diurnal anurans that inhabit the marginal rocks of streams. Juveniles of $B$. jararaca hold the prey after the bite, and use parts of the body to subjugate it. The frequent use of streams seems to be related to prey availability, and the use of vegetation at night may protect juveniles from terrestrial predators. Considering that some individuals were resting on the vegetation at night we can infer that juveniles of $B$. jararaca forage mainly on the ground during the day at the study site. Juveniles of $B$. jararacussu also use stream margins during the day to search for prey and apparently, similar to juveniles of $B$. jararaca, forage at times and places where frogs are active.
\end{abstract}

Keywords: Serpentes,Viperidae, Bothrops jararaca, Bothrops jararacussu, diet, habitat use, Atlantic Forest, southeastern Brazil.

Palavras-chave: Serpentes, Viperidae, Bothrops jararaca, Bothrops jararacussu, dieta, uso do hábitat, Mata Atlântica, sudeste do Brasil.

\section{Introdução}

A dieta é uma das principais dimensões do nicho de uma serpente e pode influenciar o uso de hábitat, seu comportamento alimentar e seu período de atividade (Toft 1985). Espécies com

Recebido em 7 de maio de 2003

Aceito em 20 de agosto de 2003

Distribuído em 31 de outubro de 2003 ampla distribuição geográfica podem apresentar grande heterogeneidade no hábito alimentar (Shine et al. 1998). Essa heterogeneidade está relacionada principalmente às diferentes disponibilidades de presas de cada região (Luiselli e Agrimi 1991, Capizzi e Luselli 1997, Shine et al. 1998). Dessa forma, especializações ou adaptações locais podem ocorrer em populações de espécies amplamente distribuídas, em função da disponibilidade e dos hábitos de suas presas 
em diferentes regiões. Em espécies com variações ontogenéticas na dieta, essas diferenças podem estar restritas a apenas uma fase da vida.

Bothrops jararaca Wied, 1824 tem distribuição ampla e associada ao domínio morfoclimático da Mata Atlântica (Ab'Saber 1977, Vanzolini 1988), estendendo-se por ambientes florestais e áreas antrópicas (Sazima 1988, 1992, Campbell e Lamar 1989). Possui atividade predominantemente noturna e mais intensa na estação chuvosa (Sazima 1992). Os juvenis de $B$. jararaca utilizam frequientemente a vegetação e apresam principalmente anuros, enquanto os adultos são predominantemente terrestres e alimentam-se basicamente de roedores (Sazima 1992, Sazima e Haddad 1992). As poucas informações disponíveis sobre Bothrops jararacussu Lacerda, 1884 indicam que essa espécie é mais restrita às áreas florestadas, raramente utiliza a vegetação e apresenta mudança ontogenética na dieta (Marques 1998).

Juvenis de $B$. jararaca e B. jararacussu podem ser encontrados com relativa facilidade em córregos da encosta da Mata Atlântica no litoral norte de São Paulo (obs. pess.). Esses córregos são ocupados por diversas espécies de anfíbios anuros, ativos durante o dia ou à noite, na vegetação, em pedras ou margens dos corpos d'água (e.g. Haddad e Giaretta 1999, Hatano et al. 2002). No presente estudo, procuramos responder às seguintes perguntas: (1) existe relação entre as espécies de anuros que ocupam os córregos e a dieta dos juvenis de $B$. jararaca?; (2) o período de atividade e o uso do substrato dos juvenis de $B$. jararaca estão relacionados aos de suas presas? Informações adicionais sobre dieta e o uso do substrato por juvenis de B. jararacussu são fornecidas e utilizadas para comparação.

\section{Material e Métodos}

O estudo foi realizado em região de Mata Atlântica no Núcleo Picinguaba do Parque Estadual da Serra do Mar, no município de Ubatuba, estado de São Paulo (2323's, $\left.44^{\circ} 50^{\prime} \mathrm{W}\right)$. As observações foram realizadas de novembro de 2001 a dezembro de 2002, em quatro córregos na encosta da serra. Esses córregos apresentam porções de água corrente intercaladas por pequenos poços, onde a água é represada, formando remansos. As margens são formadas por lajes de pedra, parcialmente sombreadas por vegetação de pequeno porte (samambaias e bambus), seguida da mata de encosta. A inclinação dos córregos varia de 20 a $40^{\circ}$, a largura varia entre 2 e $5 \mathrm{~m}$ e a altitude, entre 20 e $180 \mathrm{~m}$.

As observações foram feitas mensalmente, observando-se todo o ambiente e utilizando-se a procura limitada por tempo, tanto durante o dia como à noite (Martins e Oliveira 1998), totalizando 113 horas. Sempre que possível, os indivíduos encontrados foram observados por 30 minutos antes de qualquer interferência voluntária, a fim de verificar possíveis eventos de predação. Cada indivíduo foi fotografado, marcado por meio de picotamento das escamas ventrais (Spellerberg 1977) e teve seu comprimento rostro-cloacal (CRC) medido com o auxílio de fita métrica flexível e precisão de $1 \mathrm{~mm}$, sendo solto no mesmo local do encontro. As seguintes informações foram registradas: horário do encontro, substrato utilizado e atividade, inferida a partir da postura de cada animal observado (ver Oliveira e Martins 2002 para a descrição do método). Indivíduos com conteúdo alimentar evidente (verificado por meio de apalpação) foram coletados. Esses espécimes foram dissecados, por meio de incisão ventral ao longo dos dois terços posteriores do corpo, tendo sido obtidas as seguintes informações: conteúdo do tubo digestivo e maturidade reprodutiva. Todos os exemplares coletados foram depositados na coleção herpetológica do Instituto Butantan (IB). Para a observação do comportamento de captura e subjugação de presas, foram realizados oito encontros provocados (Sazima 1989) utilizando presas naturais (Hylodes asper ou H. phyllodes; Hylodinae, Leptodactylidae). 


\section{Resultados}

A taxa de encontro nos córregos foi de uma serpente a cada 2,89 horas de procura ou 0,34 serpente por hora-homem de procura, totalizando 39 registros. Além dessas, oito serpentes foram registradas por encontro ocasional (i.e. quando o tempo não estava sendo computado). Todos os espécimes de B. jararaca apresentavam CRC $=525 \mathrm{~mm}(\overline{\mathrm{x}}=345,6$ $\pm 79,8 ; 225-525 ; \mathrm{n}=47$ ) e foram considerados imaturos (Sazima 1992). As serpentes foram encontradas enrodilhadas, no chão ou na vegetação, ou deslocando-se nas margens dos córregos (Tabela 1). Um indivíduo recém-morto, com marcas na porção posterior da cabeça, foi encontrado no chão às 20:00 h. Os indiví- duos encontrados no chão estavam junto ao córrego, sobre pedras ou em fendas de pedras próximo aos remansos, expostos ou parcialmente abrigados sob vegetação arbustiva (Figura 1). Os indivíduos encontrados sobre a vegetação da margem dos córregos estavam enrodilhados sobre galhos finos (Figura 1) entre 20 e $160 \mathrm{~cm}$ de altura $(\overline{\mathrm{x}}=76,5 \pm 43,6)$. Durante a noite, foram encontrados indivíduos no chão $(n=6$, sendo quatro em deslocamento) e sobre a vegetação $(\mathrm{n}=8)$. Dentre os 32 indivíduos avistados durante o dia, apenas um estava sobre a vegetação.

Dentre os 16 indivíduos marcados (entre março e dezembro de 2002), oito foram reencontrados (Tabela 2). Dezoito indivíduos de $B$. jararaca foram coletados e 14 apresentavam

Tabela 1 - Número de juvenis de Bothrops jararaca encontrados ativos ou repousando no chão ou sobre a vegetação, nos córregos da encosta da Mata Atlântica. Entre parênteses, acha-se o número de indivíduos encontrados deslocando-se. Picinguaba, Ubatuba, SP.

\begin{tabular}{ccccc}
\hline & Chão & & \multicolumn{2}{c}{ Sobre a vegetação } \\
\hline Ativo & Repouso & Ativo & Repouso \\
\hline $19(6)$ & 18 & 2 & 7 \\
\hline
\end{tabular}
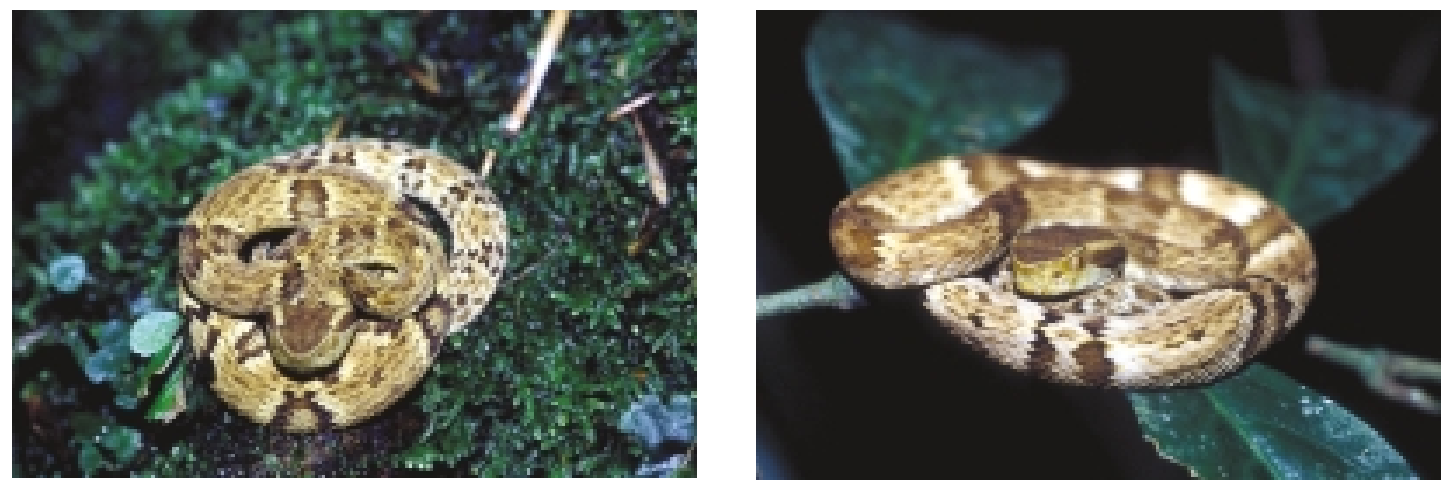

Figura 1 - Duas situações em que foram encontrados juvenis de Bothrops jararaca nas margens dos córregos: A - indivíduo ativo $(\mathrm{CRC}=355 \mathrm{~mm})$, em postura de espreita (com região anterior do corpo em $\mathrm{S}$ e inclinação da cabeça $>20^{\circ}$ em relação ao substrato) sobre pedras, durante a tarde; $\mathrm{B}$ - indivíduo em repouso $(\mathrm{CRC}=380$ $\mathrm{mm}$, cabeça não inclinada) sobre a vegetação $(85 \mathrm{~cm}$ de altura) durante a noite. Picinguaba, Ubatuba, SP. 
Tabela 2 - Data de captura e de recaptura e distância entre os pontos dos encontros dos juvenis de Bothrops jararaca marcados. Todos os indivíduos foram recapturados no mesmo córrego do primeiro encontro. Picinguaba, Ubatuba, SP.

\begin{tabular}{ccc}
\hline Data de captura & Data de recaptura & Distância entre os pontos (m) \\
\hline $15 / 05 / 2002$ & $18 / 06 / 2002$ & $70 \mathrm{~m}$ \\
$15 / 05 / 2002$ & $19 / 06 / 2002$ & $20 \mathrm{~m}$ \\
$18 / 06 / 2002$ & $20 / 06 / 2002$ & Mesmo local \\
$19 / 06 / 2002$ & $14 / 10 / 2002$ & $140 \mathrm{~m}$ \\
$19 / 08 / 2002$ & $23 / 08 / 2002$ & $20 \mathrm{~m}$ \\
$12 / 10 / 2002$ & $18 / 10 / 2002$ & $10 \mathrm{~m}$ \\
$08 / 11 / 2002$ & $15 / 11 / 2002$ & $07 \mathrm{~m}$ \\
$08 / 11 / 2002$ & $06 / 12 / 2002$ & $08 \mathrm{~m}$ \\
\hline
\end{tabular}

conteúdo no estômago. Somente anuros foram consumidos, sendo possível identificar os seguintes itens: Hylodes asper $(\mathrm{n}=3), H$. phyllodes $(\mathrm{n}=3)$, Hylodes sp. $(\mathrm{n}=2)$, Cycloramphus boraceiensis $(\mathrm{n}=1)$ e Physalaemus $\mathrm{sp}$. $(\mathrm{n}=1)$, todos pertencentes à família Leptodactylidae, além de vestígios de quatro anfíbios anuros.

Em três encontros durante o dia e um encontro noturno, os juvenis de $B$. jararaca estavam realizando engodo caudal (Sazima 1991, Hartmann e Almeida 2001). Dos encontros provocados $(n=8)$, seis juvenis capturaram a presa oferecida. Além dessas observações, foram registrados dois eventos naturais de predação de anuros pelos juvenis de B. jararaca.

O comportamento de captura e subjugação foi semelhante em todas as situações: a serpente estava enrodilhada sobre pedra na margem do córrego, durante o dia, em postura de espreita, próximo a locais utilizados por indivíduos de Hylodes asper e H. phyllodes. Quando o anuro se aproximava da serpente, esta desferia o bote, segurando a presa com a boca. Em seis observações, os juvenis pressionaram a presa contra o substrato utilizando a porção média do corpo (Figura 2). À medida que o anuro se debatia, a serpente forçava-o mais contra o substrato. Assim que o anuro cessava a resistência, a serpente

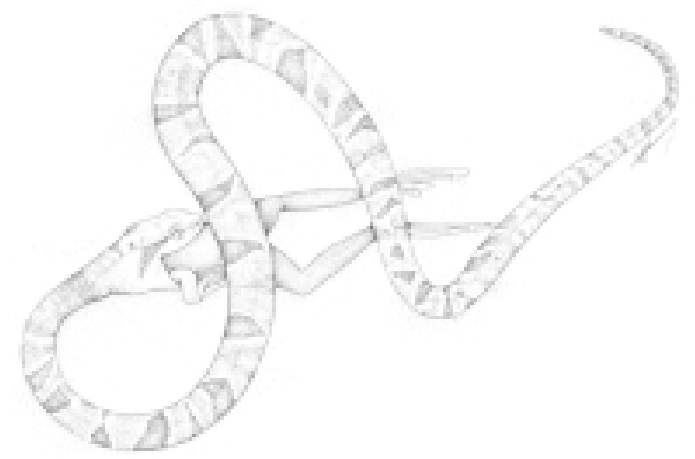

Figura 2 - Comportamento de subjugação da presa em juvenis de Bothrops jararaca, evidenciando a parte do corpo que utiliza para pressionar a presa contra o substrato. Picinguaba, Ubatuba, SP.

começava a ingerí-lo, o que ocorreu geralmente pela cabeça $(n=7)$. Após a ingestão, a serpente enrodilhava-se, em postura de repouso, no mesmo local.

Juvenis de outra espécie de viperídeo, Bothrops jararacussu $(\mathrm{CRC}=645 \mathrm{~mm} ; \mathrm{X}=$ 471,2 $\pm 123,9 ; 290-645 ; \mathrm{n}=10$ ), também foram encontrados associados aos córregos na mata de encosta. Todos foram encontrados durante o dia e no chão, enrodilhados sobre pedras nas margens dos córregos. Destes, oito estavam 
ativos e dois achavam-se em repouso. Três apresentavam presas no estômago, todas anuros (dois Hylodes asper e um anuro não-identificado). Um indivíduo foi encontrado ingerindo um anuro (H. asper).

\section{Discussão}

A freqüência de encontros de juvenis de Bothrops jararaca nas encostas da Serra do Mar de Picinguaba foi alta quando comparada à de outros estudos (Marques 1998, Sazima 1988). Isso provavelmente se deve ao tipo de hábitat (córregos) explorado neste estudo. O uso do hábitat por determinada espécie de serpente parece estar relacionado à abundância das presas (Reinert 1993). Juvenis de B. jararaca alimentam-se principalmente de anuros (Sazima 1992), e os córregos estudados têm maior disponibilidade de anuros em comparação com outras regiões de mata. Três espécies de anuros podem ser encontradas ativas nesses córregos durante o dia, nas pedras ou nas margens dos córregos: Hylodes asper, H. phyllodes e Cycloramphus boraceiensis (Leptodoctylidae). À noite, diversas espécies de anuros são encontradas sobre a vegetação, como os hilídeos Scinax trapicheiroi, Phasmahyla guttata e Hyla sp. (aff. circumdata) (obs. pess.). Durante à noite, Thoropa miliaris e $C$. boraceiensis podem utilizar as lajes de pedras dos córregos, enquanto Physalaemus sp. e Eleutherodactylus binotatus (Leptodactylidae) ocupam o chão da mata marginal aos córregos.

A utilização de determinado micro-hábitat parece estar relacionada à atividade da serpente em cada período (Reinert 1993). Por exemplo, quando repousando, a serpente pode utilizar locais que confiram maior segurança contra a predação (Martins 1993) e, quando forrageando, locais de maior probabilidade de encontro da presa (Bernarde et al. 2000, Hartmann 2001). O uso mais freqüente da vegetação durante a noite pelos juvenis de $B$. jararaca pode estar relacionado à proteção contra predadores noturnos, principalmente peque- nos marsupiais (Lutreolina crassicaudata, Philander opossum e Didelphis marsupialis) (Sazima 1992, Oliveira e Santori 1999). O indivíduo encontrado morto estava no chão, com sinais de predação provavelmente causados por mordidas de marsupiais. Esses predadores geralmente utilizam o chão para forragear (Emmons 1990). A ausência de anuros arborícolas na dieta de juvenis de $B$. jararaca indica que a atividade de forrageamento dessas serpentes deve estar restrita ao chão.

Algumas espécies de serpentes que se alimentam de anuros forrageiam durante a noite, por ser este o período em que as presas estão em atividade (Vitt 1996, Martins e Oliveira 1998, Bernarde et al. 2000). Da mesma forma, juvenis de $B$. jararaca - que caçam por espreita - capturam presas ativas, que, nos córregos, podem ser encontradas em qualquer horário. Porém, as presas consumidas pelos filhotes de $B$. jararaca são espécies de atividade diurna. Além disso, se considerarmos que mais da metade dos indivíduos encontrados à noite estavam em repouso sobre a vegetação, pode-se inferir que esses juvenis forrageiam principalmente no chão e durante o dia.

Os juvenis de $B$. jararaca permanecem nos córregos e arredores por determinado tempo, como indica a recaptura de indivíduos meses após o primeiro encontro. Os filhotes utilizam os córregos para caçar, em função da abundância de presas. Com o crescimento, os indivíduos de $B$. jararaca possivelmente passam a utilizar outros sítios de forrageio, devido à variação ontogenética da dieta da espécie (Sazima 1992).

O comportamento de subjugação, que envolve o pressionamento da presa contra o substrato com a porção média do corpo, não havia sido registrado anteriormente (Sazima 1992). Esse comportamento foi comparado com a definição de constrição apresentada por Greene e Burghardt (1978). Os juvenis de $B$. jararaca não enlaçaram a presa, e houve poucos pontos de contato entre o corpo da serpente e a presa (no máximo dois). No entanto, pres- 
sionaram a presa contra o substrato, que serviu como ponto de apoio, o que permite classificar tal modo de subjugação como constrição.

Anfíbios anuros são presas comuns para serpentes sulamericanas, incluindo viperídeos (Vitt e Vangilder 1983, Duellman 1990, Strüssmann e Sazima 1993, Martins et al. 2002). Esse tipo de presa oferece poucos riscos de retaliação ao predador e pode ser seguro pela serpente com relativa segurança. Entretanto, se for solto, pode distanciar-se da serpente aos saltos, deixando trilha química pouco perceptível. Nos córregos estudados, essa situação é mais acentuada, pois, se o anuro cair na água, poderá ser levado pela correnteza, com poucas chances de ser encontrado e ingerido (obs. pess.). Dessa forma, é possível que o comportamento de subjugação dos juvenis de $B$. jararaca, pressionando o anuro contra o substrato, seja uma forma de diminuir as possibilidades de fuga da presa.

Os juvenis de $B$. jararacussu utilizam as margens dos córregos durante o dia para caçar, possivelmente aproveitando-se da abundância de anuros desse ambiente. Porém, durante a noite, não foram encontrados nos córregos e possivelmente refugiem-se dentro da mata. Dessa forma, assim como os juvenis de $B$. jararaca, o local e o período de forrageamento dos juvenis de $B$. jararacussu parecem estar relacionados ao substrato e ao período de atividade das presas nos córregos da Mata Atlântica.

\section{Agradecimentos}

Agradecemos a C. F. B. Haddad, I. Sazima, L. J. Vitt, M. R. C. Martins e O. A. V. Marques pelas discussões e valiosos comentários sobre o manuscrito. Aos funcionários do Núcleo Picinguaba do Parque Estadual da Serra do Mar pelo apoio durante a permanência nas instalações. Ao Instituto Brasileiro do Meio Ambiente e Recursos Naturais Renováveis - IBAMA pela licença de captura e coleta $\left(n^{\circ} 37 / 2001\right)$. Ao CNPq, Capes e Fapesp pelo fornecimento de bolsas e auxílio financeiro.

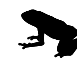

\section{Referências Bibliográficas}

Ab'Saber, J. W. 1977. Os domínios morfoclimáticos na América do sul. Primeira aproximação. Geomorfologia 52: $1-21$.

Bernarde, P. S., M. N. C. Kokubum e O. A. V., Marques. 2000. Utilização de hábitat e atividade em Thamnodynastes strigatus (Günther, 1858) no sul do Brasil (Serpentes, Colubridae). Boletim do Museu Nacional, Nova Série, Zoologia 428: 1-8.

Campbell, J. A. e W. Lamar. 1989. The Venomous Reptiles of Latin America. Ithaca. Cornell University Press.

Capizzi, D. e L. Luiselli. 1997. The diet of the four-lined (Elaphe quatuorlineata) in Mediterranean central Italy. Herpetological Journal 7: 1-5.

Duellman, W. E. 1990. Herpetofauna in neotropical rainforests: comparative composition, history, and resource use. Pp. 455-505 in A. H. Gentry (ed.), Four Neotropical Rainforests. New Haven. Yale University Press.

Emmons, L. H. 1990. Neotropical Rainforest Mammals. Chicago. The University of Chicago Press. 281 pp.

Greene, H. e M. Burghardt. 1978. Behavior and phylogeny: constriction in ancient and modern snakes. Science 200: 74-77.

Haddad, C. F. B. e A. A. Giaretta. 1999. Visual and acoustic communication in the Brazilian Torrent Frog, Hylodes asper (Anura: Leptodactylidae). Herpetologica 55: 324-333.

Hartmann, P. A. 2001. Hábito alimentar e utilização do ambiente em duas espécies simpátricas de Philodryas (Serpentes, Colubridae) no sul do Brasil. Dissertação de Mestrado Não-publicada. Universidade Estadual Paulista, Brasil.

Hartmann, P. A. e M. T. Almeida. 2001. Bothrops jararaca (Jararaca pitviper). Caudal luring. Herpetological Review 32: 45.

Hatano, F. H., C. F. Rocha e M. V. Sluys. 2002. Enviromental factors affecting calling activity of a tropical diurnal frog (Hylodes phyllodes: Leptodactylidae). Journal of Herpetology 36: 314-318.

Luiselli, L. e U. Agrimi. 1991. Composition and variation of diet of Vipera aspis francisciredi in relation to age and reproductive stage. Amphibia-Reptilia 12: 137144.

Marques, O. A. V. 1998. Composição faunística, história natural e ecologia de serpentes da mata atlântica, na região da estação ecológica Juréia-Itatins, São Paulo, SP. Tese de Doutorado Não-publicada. Universidade de São Paulo, Brasil.

Martins, M. R. C. 1993. Why do snakes sleep on the vegetation in central Amazônia. Herpetological 
Review 24: 83-84.

Martins, M. R. C. e M. E. Oliveira. 1998. Natural history of snakes in forest of the Manaus, central Amazonia, Brazil. Herpetological Natural History 6: 78-150.

Martins, M., O. A. V. Marques e I. Sazima. 2002. Ecological and phylogenetic correlates of feeding habits in Neotropical pitvipers of the genus Bothrops. Pp. 307-328 in G. Schuett, M. Höggren e H. W. Greene (eds.), Biology of the Vipers. Carmel, Indiana. Biological Sciences Press.

Oliveira, M. E. e M. R. C. Martins. 2002. When and where to find a pitviper: activity patterns and habitat use of the lancehead, Bothrops atrox, in central Amazonia, Brazil. Herpetological Natural History 8: 101-110.

Oliveira, M. E. e R. T. Santori. 1999. Predatory behavior of Opossum Didelphis albiventris on the Pitviper Bothrops jararaca. Studies on Neotropical Fauna and Environment 34: 72-75.

Reinert, H. K. 1993. Habitat selection in snakes. Pp. 201240 in R. A. Seigel e J. T. Collins (eds.), Snakes ecology and behavior. New York. McGraw-Hill.

Sazima, I. 1988. Um estudo de biologia comportamental da jararaca, Bothrops jararaca,com uso de marcas naturais. Memórias do Instituto Butantan 50: 83-90.

Sazima, I. 1989. Comportamento alimentar da jararaca, Bothrops jararaca: encontros provocados na natureza. Ciência e Cultura 41: 500-505.

Sazima, I. 1991. Caudal luring in two Neotropical pitvipers, Bothrops jararaca and Bothrops jararacussu. Copeia 1991: 245-248.

Sazima, I. 1992. Natural history of jararaca pitviper, Bothrops jararaca in southeastern Brazil. Pp.199-216 in J. A. Campbell e E. D. Brodie Jr. (eds.), Biology of the Pitvipers, Tyler (Texas). Selva.

Sazima, I. e C. F. B. Haddad. 1992. Répteis da Serra do Japi: notas sobre história natural. Pp. 212-237 in L. P. Morellato (ed.). História Natural da Serra do Japi - ecologia e preservação de uma área florestal no sudeste do Brasil. Campinas. FAPESP.

Shine, R., W. R. Branch, P. S. Harlow e J. K. Webb. 1998. Reproductive biology and food habits of horned adders, Bitis caudalis (Viperidae), from Southern Africa. Copeia 1998: 391-401.

Spellerberg, I. F. 1977. Making live snakes for identification of individuals on population studies. Journal of Application Ecology 14: 137-138.

Strüssmann, C. e I. Sazima. 1993. The snake assemblage of the pantanal at Poconé, western Brazil: faunal composition and ecological summary. Studies on Neotropical Fauna and Environment 28: 157-168.

Toft, C. A. 1985. Resource partitioning in amphibians and reptiles. Copeia 1985: 1-21.

Vanzolini. P. E. 1988. Distributional patterns of South American lizards. Pp. 317-342 in P. E. Vanzolini e W. R. Heyer (eds.), Proceedings of a Workshop on Neotropical Distribution Patterns. Rio de Janeiro. Academia Brasileira de Ciências.

Vitt, L. J. 1996. Ecological observations on the tropical colubrid snake Leptodeira annulata. Herpetological Natural History 4: 69-76.

Vitt, L. J. e L. D. Vangilder. 1983. Ecology of a snake community in northeastern Brazil. Amphibia-Reptilia 4: 273-296. 